This item was submitted to Loughborough's Research Repository by the author.

Items in Figshare are protected by copyright, with all rights reserved, unless otherwise indicated.

\title{
Nevanlinna theory for the q-difference operator and meromorphic solutions of q-difference equations
}

PLEASE CITE THE PUBLISHED VERSION

LICENCE

CC BY-NC-ND 4.0

\section{REPOSITORY RECORD}

Barnett, David C., R.G. Halburd, R.J. Korhonen, and W. Morgan. 2019. "Nevanlinna Theory for the Qdifference Operator and Meromorphic Solutions of Q-difference Equations". figshare. https://hdl.handle.net/2134/769. 


\title{
Nevanlinna theory for the $q$-difference operator and meromorphic solutions of $q$-difference equations
}

\author{
D. C. Barnett, R. G. Halburd, R. J. Korhonen`and W. Morgan*
}

February 6, 2006

\begin{abstract}
It is shown that, if $f$ is a meromorphic function of order zero and $q \in \mathbb{C}$, then

$$
m\left(r, \frac{f(q z)}{f(z)}\right)=o(T(r, f))
$$

for all $r$ on a set of logarithmic density 1 . The remainder of the paper consist of applications of identity $(\ddagger)$ to the study of value distribution of zero-order meromorphic functions, and, in particular, zero-order meromorphic solutions of $q$-difference equations. The results obtained include $q$-shift analogues of the Second Main Theorem of Nevanlinna theory, Picard's theorem, and Clunie and Mohon'ko lemmas.
\end{abstract}

\section{Introduction}

Valiron has shown that the non-autonomous Schröder $q$-difference equation

$$
f(q z)=R(z, f(z))
$$

where $R(z, f(z))$ is rational in both arguments, admits a one parameter family of meromorphic solutions, provided that $q \in \mathbb{C}$ is chosen appropriately [27]. It was shown by Gundersen et al. [10] that the order of growth of these solutions is equal to $\log _{q}\left(\operatorname{deg}_{f} R\right)$, where $\log _{q}$ is the $q$-based logarithm. Their result implies a $q$-difference analogue of the classical Malmquist's theorem [23]: if the $q$-difference equation (1) admits a meromorphic solution of order zero, then (1) reduces to a $q$-difference Riccati equation, i.e. $\operatorname{deg}_{f} R=1$.

\footnotetext{
*Permanent address: Department of Mathematical Sciences, Loughborough University, Loughborough, Leicestershire, LE11 3TU, UK (d.c.barnett@lboro.ac.uk, r.g.halburd@lboro.ac.uk, w.morgan@lboro.ac.uk)

†Permanent address: Department of Mathematics, University of Joensuu, P.O. Box 111, FI-80101 Joensuu, Finland (risto.korhonen@joensuu.fi)

Mathematics Subject Classification 2000: Primary 30D35; Secondary 39A13, 39B32, 30D05.
} 
There are a variety of methods which can be used to study the value distribution of meromorphic solutions of the Schröder equation (1). Eremenko and Sodin [8] used methods from complex dynamics to show that the Valiron and Nevanlinna deficient values of meromorphic solutions of the autonomous Schröder equation (1) always coincide with the exceptional values of $R(z)$. Ishizaki and Yanagihara [18] constructed an example showing that this is not true in general for the non-autonomous Schröder equation. They have also applied Nevanlinna theory to study Borel and Julia directions of meromorphic solutions of the Schröder equation $[19,20]$.

Linear $q$-difference equations with rational coefficients do not always admit meromorphic solutions, even if the coefficients are constants. Bergweiler, Ishizaki and Yanagihara gave sufficient conditions for the existence of meromorphic solutions of linear $q$-difference equations, and characterized the growth of solutions in terms of the Nevanlinna characteristic $T(r, f)[3]$. They concluded that all meromorphic solutions $f$ of a linear $q$-difference equation with rational coefficients satisfy $T(r, f)=O\left((\log r)^{2}\right)$, from which it in particular follows that all solutions are of zero order of growth. For entire solutions there are efficient methods, for instance, a $q$-shift analogue of the Wiman-Valiron theory by Bergweiler, Ishizaki and Yanagihara [4], and a functional analytic method by Ramis [26], by which the asymptotic behavior of solutions may be characterized even more precisely.

Bergweiler and Hayman [2] studied the zero distribution of entire solutions of linear $q$-difference equations. They found an asymptotic formula for the locations of zeros of solutions by showing that, under a certain condition, solutions behave asymptotically like products of $\theta$-functions. Heittokangas et al. [17] studied the density of zeros and poles of meromorphic solutions of linear $q$-difference equations by using methods from Nevanlinna theory.

When applying Nevanlinna theory to study the growth and value distribution of meromorphic solutions of differential equations, estimates involving logarithmic derivatives have often proved to be useful $[9,22]$. Recently, similar tools involving shifts have been developed to study ordinary difference equations $[6,13,14]$. The following theorem [13] is among the fundamental results of this type.

Theorem A Let $f$ be a non-constant finite-order meromorphic function, and $c \in \mathbb{C}$. Then

$$
m\left(r, \frac{f(z+c)}{f(z)}\right)=o\left(\frac{T(r, f)}{r^{\delta}}\right)
$$

for any $\delta<1$, and for all $r$ outside of an exceptional set with finite logarithmic measure.

Theorem A and its corollaries proved to be indispensable when singling out Painlevé type equations out of large classes of difference equations $[11,12]$. Theorem A may also be used to study value distribution of finite-order meromorphic solutions of large classes of difference equations, including difference Riccati and difference Painlevé equations. 
One of the main results of this paper is the following theorem which is a $q$-difference analogue of Theorem A, and of the Lemma on the Logarithmic Derivative.

Theorem 1.1 Let $f$ be a non-constant zero-order meromorphic function, and $q \in \mathbb{C} \backslash\{0\}$. Then

$$
m\left(r, \frac{f(q z)}{f(z)}\right)=o(T(r, f))
$$

on a set of logarithmic density 1 .

Theorem 1.1 may be used to study zero-order meromorphic solutions of $q$ difference equations in a similar manner as Theorem A applies for finite-order meromorphic solutions of difference equations. The restriction to zero-order meromorphic functions is analogous to demanding finite order of growth in the ordinary shift case. For instance, all meromorphic solutions of linear and $q$ Riccati difference equation are of zero order.

Concerning the sharpness of Theorem 1.1, the exponential function does not satisfy (2) for any $q \in \mathbb{C}$, and so the assertion of Theorem 1.1 cannot be extended to hold for all finite-order meromorphic functions.

The remainder of the paper is organized as follows. Before proving Theorem 1.1 we discuss its applications to $q$-difference equations in Section 2. We present $q$-shift analogues of the Clunie and Mohon'ko lemmas which can be used to study value distribution of zero-order meromorphic solutions of large classes of $q$-difference equations. In Section 3 , we prove a $q$-shift analogue of the Second Main Theorem of Nevanlinna theory, and give an outline of how it can be used to analyze the value distribution of zero-order meromorphic functions. Its corollaries include a Picard type theorem for the $q$-shift operator. We give explicit examples of $q$-difference equations with zero-order meromorphic solutions expressible in terms of $q$-Gamma functions in Section 4. These examples show that the results obtained in Sections 2 and 3 are, in a sense, best possible. In Section 5 we prove Theorem 1.1 by a series of lemmas, and finally, we make some concluding remarks concerning our results in Section 6.

\section{Applications to $q$-Difference Equations}

Theorem 1.1 is a powerful tool in the study of complex analytic properties of zero-order meromorphic solutions of large classes of $q$-difference equations. In this section we are concerned with functions which are polynomials in $f\left(q_{j} z\right)$, where $q_{j} \in \mathbb{C}$, with coefficients $a_{\lambda}(z)$ such that

$$
T\left(r, a_{\lambda}(z)\right)=o(T(r, f))
$$

on a set of logarithmic density 1 . Such functions will be called $q$-difference polynomials in $f(z)$.

The following theorem is analogous to the Clunie Lemma [7]. It can be used to study pole distribution of meromorphic zero-order solutions of non-linear $q$-difference equations. 
Theorem 2.1 Let $f(z)$ be a non-constant zero-order meromorphic solution of

$$
f(z)^{n} P(z, f)=Q(z, f),
$$

where $P(z, f)$ and $Q(z, f)$ are $q$-difference polynomials in $f(z)$. If the degree of $Q(z, f)$ as a polynomial in $f(z)$ and its $q$-shifts is at most $n$, then

$$
m(r, P(z, f))=o(T(r, f))
$$

on a set of logarithmic density 1.

Proof. We follow the reasoning behind the original Clunie Lemma, see, for instance, [22], replacing the Lemma on the Logarithmic Derivative with Theorem 1.1.

In calculating the proximity function of $P$, we split the region of integration into two parts. By defining

$$
E_{1}:=\left\{\varphi \in[0,2 \pi]:\left|f\left(r e^{i \varphi}\right)\right|<1\right\}
$$

and

$$
E_{2}:=[0,2 \pi] \backslash E_{1},
$$

we have

$$
2 \pi m(r, P(z, f))=\int_{E_{1}} \log ^{+}|P| d \varphi+\int_{E_{2}} \log ^{+}|P| d \varphi .
$$

First we consider $E_{1}$. Each term of $P$ is of the form

$$
a_{\lambda}(z) f(z)^{l_{0}} f\left(q_{1} z\right)^{l_{1}} \cdots f\left(q_{\nu} z\right)^{l_{\nu}},
$$

and so, writing with $\lambda=\left(l_{0}, \ldots, l_{\nu}\right)$,

$$
P(z, f)=\sum_{\lambda \in I} P_{\lambda}(z, f)=\sum_{\lambda \in I} a_{\lambda}(z) f(z)^{l_{0}} f\left(q_{1} z\right)^{l_{1}} \cdots f\left(q_{\nu} z\right)^{l_{\nu}} .
$$

For each $\lambda$ we have

$$
\left|P_{\lambda}\left(r e^{i \varphi}\right)\right| \leq\left|a_{\lambda}\left(r e^{i \varphi}\right)\right|\left|\frac{f\left(q_{1} r e^{i \varphi}\right)}{f\left(r e^{i \varphi}\right)}\right|^{l_{1}} \ldots\left|\frac{f\left(q_{\nu} r e^{i \varphi}\right)}{f\left(r e^{i \varphi}\right)}\right|^{l_{\nu}},
$$

whenever $\varphi \in E_{1}$. Therefore for each $\lambda$ we obtain

$$
\int_{E_{1}} \log ^{+}\left|P_{\lambda}\left(r e^{i \varphi}\right)\right| \frac{d \varphi}{2 \pi} \leq m\left(r, a_{\lambda}\right)+O\left(\sum_{j=1}^{\nu} m\left(r, \frac{f\left(q_{j} z\right)}{f(z)}\right)\right),
$$

which, together with Theorem 1.1 and our assumption, implies that

$$
\int_{E_{1}} \log ^{+}\left|P\left(r e^{i \varphi}, f\right)\right| \frac{d \varphi}{2 \pi}=o(T(r, f))
$$


on a set of logarithmic density 1 .

Now we consider $E_{2}$. To do this case we note that

$$
Q(z, f)=\sum_{\gamma \in J} Q_{\gamma}(z, f)=\sum_{\gamma \in J} b_{\gamma}(z) f\left(q_{0} z\right)^{l_{0}} \cdots f\left(q_{\mu} z\right)^{l_{\mu}},
$$

where, by our assumption $l_{1}+\cdots+l_{\mu} \leq n$ for all $\gamma=\left(l_{0}, \ldots, l_{\mu}\right) \in J$. Hence we have

$$
\begin{aligned}
|P(z, f)| & =\left|\frac{1}{f(z)^{n}} \sum_{\gamma \in J} b_{\gamma}(z) f(z)^{l_{0}} f\left(q_{1} z\right)^{l_{1}} \cdots f\left(q_{\mu} z\right)^{l_{\mu}}\right| \\
& \leq \sum_{\gamma \in J}\left|b_{\gamma}(z)\right|\left|\frac{f\left(q_{1} r e^{i \varphi}\right)}{f\left(r e^{i \varphi}\right)}\right|^{l_{1}} \cdots\left|\frac{f\left(q_{\mu} r e^{i \varphi}\right)}{f\left(r e^{i \varphi}\right)}\right|^{l_{\mu}}
\end{aligned}
$$

and so, by Theorem 1.1 again,

$$
\int_{E_{2}} \log ^{+}\left|P\left(r e^{i \varphi}, f\right)\right| \frac{d \varphi}{2 \pi}=o(T(r, f))
$$

on a set of logarithmic density 1 . The assertion follows by combining (3), (4) and (5).

Let $\alpha$ and $f$ be meromorphic zero-order functions such that $T(r, \alpha)=$ $o(T(r, f))$ on a set of logarithmic density 1 . Then $\alpha$ is called a slowly moving target or a small function with respect to $f$. In particular, constant functions are always slowly moving compared to any non-constant meromorphic function. The next result can be used as tool to analyze the value distribution of zeroorder meromorphic solutions $f$, with respect to slowly moving targets. It is an analogue of a result due to A. Z. Mohon'ko and V. D. Mohon'ko [24] on differential equations.

Theorem 2.2 Let $f(z)$ be a non-constant zero-order meromorphic solution of

$$
P(z, f)=0
$$

where $P(z, f)$ is a q-difference polynomial in $f(z)$. If $P(z, \alpha) \not \equiv 0$ for slowly moving target $\alpha$, then

$$
m\left(r, \frac{1}{f-\alpha}\right)=o(T(r, f))
$$

on a set of logarithmic density 1.

Proof. By substituting $f=g+\alpha$ into (6) we obtain

$$
Q(z, g)+D(z)=0,
$$


where

$$
Q(z, g)=\sum_{\gamma=\left(j_{0}, \ldots, j_{\nu}\right) \in J} b_{\gamma}(z) g(z)^{j_{0}} g\left(q_{1} z\right)^{j_{1}} \cdots g\left(q_{\nu} z\right)^{j_{\nu}}
$$

is a $q$-difference polynomial in $g$ such that all of its terms are at least degree one, and $T(r, D)=o(T(r, g))$ on a set of logarithmic density 1 . Also $D \not \equiv 0$, since $\alpha$ does not satisfy $(6)$.

Using (7) we have

$$
\begin{aligned}
m\left(r, \frac{1}{g}\right) & \leq m\left(r, \frac{D}{g}\right)+m\left(r, \frac{1}{D}\right) \\
& =m\left(r, \frac{Q(z, g)}{g}\right)+m\left(r, \frac{1}{D}\right) .
\end{aligned}
$$

Note that since the integral $m(r, 1 / g)$ vanishes on the part of $|z|=r$ where $|g|>1$ it is sufficient to consider only the case $|g| \leq 1$ from now on. Then

$$
\begin{aligned}
\left|\frac{Q(z, g)}{g}\right| & =\frac{1}{|g|}\left|\sum_{\gamma \in J} b_{\gamma}(z) g(z)^{l_{0}} g\left(q_{1} z\right)^{l_{1}} \cdots g\left(q_{\nu} z\right)^{l_{\nu}}\right| \\
& \leq \sum_{\gamma \in J}\left|b_{\gamma}(z)\right|\left|\frac{g\left(q_{1} z\right)}{g(z)}\right|^{l_{1}} \cdots\left|\frac{g\left(q_{\nu} z\right)}{g(z)}\right|^{l_{\nu}},
\end{aligned}
$$

and

$$
m\left(r, b_{\gamma}\right)=o(T(r, g))
$$

on a set of logarithmic density 1 for all $\gamma \in J$. Also by Theorem 1.1

$$
m\left(r, \frac{g(q z)}{g(z)}\right)=o(T(r, g))
$$

on a set of logarithmic density 1 for all $q \in \mathbb{C}$. Hence by (8) and (9) and the fact that $\sum_{j=0}^{\nu} l_{j} \geq 1$ for all $\gamma \in J$ we have

$$
m\left(r, \frac{1}{g}\right)=o(T(r, g))
$$

on a set of logarithmic density 1 . Since $g=f-\alpha$ the assertion follows.

\section{Second Main Theorem}

In this section we present a $q$-shift analogue of the Second Main Theorem of Nevanlinna theory and discuss its applications in the study of the value distribution of zero-order meromorphic functions.

Let $f(z)$ be a non-constant meromorphic function of zero order, let $q \in$ $\mathbb{C} \backslash\{0,1\}$ and let $a \in \mathbb{C}$. By denoting

$$
\Delta_{q} f:=f(q z)-f(z)
$$


and by applying Theorem 1.1 with the function $f(z)-a$, we have

$$
m\left(r, \frac{\Delta_{q} f}{f-a}\right)=o(T(r, f))
$$

on a set of logarithmic density 1 . If $g$ is a meromorphic function such that $T(r, g)=o(T(r, f))$ for all $r$ on a set of logarithmic density 1, or in other words $g$ is slowly moving with respect to $f$, we say that $T(r, g)=S_{q}(r, f)$.

The following theorem is obtained by modifying Nevanlinna's proof of the Second Main Theorem [25], and by applying Theorem 1.1.

Theorem 3.1 Let $f$ be a non-constant meromorphic function of zero order, let $q \in \mathbb{C} \backslash\{0,1\}$, and let $a_{1}, \ldots, a_{p} \in \mathbb{C}, p \geq 2$, be distinct points. Then

$$
m(r, f)+\sum_{k=1}^{p} m\left(r, \frac{1}{f-a_{k}}\right) \leq 2 T(r, f)-N_{\text {pair }}(r, f)+S_{q}(r, f)
$$

where

$$
N_{\text {pair }}(r, f):=2 N(r, f)-N\left(r, \Delta_{q} f\right)+N\left(r, \frac{1}{\Delta_{q} f}\right) .
$$

Proof. Using the First Main Theorem we have

$$
\begin{aligned}
\sum_{k=1}^{p} m\left(r, \frac{1}{f-a_{k}}\right) & =\sum_{k=1}^{p} T\left(r, \frac{1}{f-a_{k}}\right)-\sum_{k=1}^{p} N\left(r, \frac{1}{f-a_{k}}\right) \\
& =p T(r, f)-N\left(r, \frac{1}{P(f)}\right)+S_{q}(r, f),
\end{aligned}
$$

where

$$
P(f)=\prod_{k=1}^{p}\left(f-a_{k}\right)
$$

Since

$$
\frac{1}{P(f)}=\sum_{k=1}^{p} \frac{\alpha_{k}}{f-a_{k}}
$$

for some constants $\alpha_{k}$, it follows by (10), that

$$
m\left(r, \frac{\Delta_{q} f}{P(f)}\right) \leq \sum_{k=1}^{p} m\left(r, \frac{\Delta_{q} f}{f-a_{k}}\right)+S_{q}(r, f)=S_{q}(r, f),
$$

and so

$$
m\left(r, \frac{1}{P(f)}\right)=m\left(r, \frac{\Delta_{q} f}{P(f)} \frac{1}{\Delta_{q} f}\right) \leq m\left(r, \frac{1}{\Delta_{q} f}\right)+S_{q}(r, f) .
$$


By the Valiron-Mohon'ko identity

$$
p T(r, f)=T(r, P(f))+O(1),
$$

and thus, by applying the First Main Theorem and inequality (12), equation (11) becomes

$$
\begin{aligned}
\sum_{k=1}^{p} m\left(r, \frac{1}{f-a_{k}}\right) & =T(r, P(f))-N\left(r, \frac{1}{P(f)}\right)+S_{q}(r, f) \\
& =m\left(r, \frac{1}{P(f)}\right)+S_{q}(r, f) \\
& \leq m\left(r, \frac{1}{\Delta_{q} f}\right)+S_{q}(r, f) \\
& =T\left(r, \Delta_{q} f\right)-N\left(r, \frac{1}{\Delta_{q} f}\right)+S_{q}(r, f) .
\end{aligned}
$$

Therefore we have

$$
\begin{aligned}
m(r, f)+\sum_{k=1}^{p} m\left(r, \frac{1}{f-a_{k}}\right) \leq & T(r, f)+N\left(r, \Delta_{q} f\right)+m\left(r, \Delta_{q} f\right) \\
& -N\left(r, \frac{1}{\Delta_{q} f}\right)-N(r, f)+S_{q}(r, f) .
\end{aligned}
$$

But

$$
m\left(r, \Delta_{q} f\right)=m\left(r, f \frac{\Delta_{q} f}{f}\right) \leq m(r, f)+m\left(r, \frac{\Delta_{q} f}{f}\right)=m(r, f)+S_{q}(r, f)
$$

by equation (10). This implies

$$
\begin{gathered}
m(r, f)+\sum_{k=1}^{p} m\left(r, \frac{1}{f-a_{k}}\right) \leq 2 T(r, f)+N\left(r, \Delta_{q} f\right)-N\left(r, \frac{1}{\Delta_{q} f}\right) \\
-2 N(r, f)+S_{q}(r, f),
\end{gathered}
$$

as required.

We will now give examples of how to apply Theorem 3.1 to analyze the value distribution of zero-order meromorphic functions. Let $a, b$ and $c$ be distinct points in the extended complex plane, and assume that $f$ is a meromorphic function of order zero such that all $a, b$ and $c$-points of $f$ appear only in infinite point sets $\left\{q^{n} z_{j}\right\}_{n \in \mathbb{N} \cup\{0\}}$, where $z_{j} \in \mathbb{C}$ is the generating point of the set. (Note that this assumption do not rule out the possibility that some, or all, of the values $a, b$ and $c$ are Picard exceptional.) Assume further that multiplicities of values of $f$ do not decrease when $n$ increases. Without loss of generality we may take $a=0, b=1$ and $c=\infty$. Then,

$$
N(r, f)+N\left(r, \frac{1}{f}\right)+N\left(r, \frac{1}{f-1}\right) \leq N_{\text {pair }}(r, f)+S_{q}(r, f),
$$


and so, by Theorem 3.1,

$$
T(r, f)=S_{q}(r, f)
$$

which is a contradiction, unless $f$ is a constant function.

We say that the value $a$ in the extended complex plane is a $q$-Picard exceptional value of a zero-order meromorphic function $f$ if $q \in \mathbb{C} \backslash\{0,1\}$, and $f$ assumes the value $a$ only in the sets $\left\{q^{n} z_{j}\right\}_{n \in \mathbb{N} \cup\{0\}}$ for some $z_{j}$, with at most finitely many exceptions. Then the above reasoning may be summarized as follows.

Corollary 3.2 If a zero-order meromorphic function $f$ has at least three $q$ Picard exceptional values, then $f$ is constant.

Corollary 3.2 may be described as a $q$-shift analogue of Picard's theorem. Concerning the sharpness of this result, zero-order meromorphic functions with two distinct $q$-Picard exceptional values can be constructed by means of Hadamard products [15]. An explicit example showing the sharpness of Corollary 3.2 can be found in the following section.

One can obtain more precise information concerning the value distribution of zero-order meromorphic functions by a careful analysis of the term $N_{\text {pair }}(r, f)$ in Theorem 3.1. In the remainder of this section we describe the relevant terminology needed towards this end and list a number of theorems which can be obtained by using Theorem 3.1 together with known methods from Nevanlinna theory. We omit the proofs of these results since one can easily reproduce them from proofs of the analogous results in [14] by using Theorem 1.1 instead of Theorem A.

Let $q \in \mathbb{C} \backslash\{0,1\}$ and $a \in \mathbb{C}$. We define the counting function $n_{q}(r, a)$ to be the number of points $z_{0}$ in the disc of radius $r$ centered at the origin such that $f\left(z_{0}\right)=a$ and $f\left(q z_{0}\right)=a$, where the contribution to $n_{q}(r, a)$ is the number of equal terms in the beginning of Taylor series expansions of $f(z)$ and $f(q z)$ in a neighborhood of $z_{0}$. We call such points $q$-separated a-pairs of $f$ in the disc $\{z:|z| \leq r\}$. The number of q-separated pole pairs $n_{q}(r, \infty)$ is the number of $q$-separated 0 -pairs of $1 / f$. This means that if $f$ has a pole with multiplicity $p$ at $z_{0}$ and another pole with multiplicity $s$ at $q z_{0}$ then this pair is counted $\min \{p, s\}+m$ times in $n_{q}(r, \infty)$, where $m$ is the number of equal terms in the beginning of the Laurent series expansions of $f(z)$ and $f(q z)$ in a neighborhood of $z_{0}$.

The integrated counting functions are defined in a natural way as

$$
N_{q}\left(r, \frac{1}{f-a}\right) \equiv N_{q}(r, a):=\int_{0}^{r} \frac{n_{q}(t, a)-n_{q}(0, a)}{t} d t+n_{q}(0, a) \log r
$$

and

$$
N_{q}(r, f) \equiv N_{q}(r, \infty):=\int_{0}^{r} \frac{n_{q}(t, \infty)-n_{q}(0, \infty)}{t} d t+n_{q}(0, \infty) \log r
$$

Then, a natural $q$-difference analogue of $\bar{N}(r, a)$ is

$$
\widetilde{N}_{q}(r, a):=N(r, a)-N_{q}(r, a)
$$


which counts the number of those $a$-points (or poles) of $f$ which are not $q$ separated pairs. Note that, unlike $\bar{N}(r, a)$, it is at least in principle possible that $\widetilde{N}_{q}(r, a)$ is negative for all $r$. The following theorem is a generalization of Corollary 3.2.

Theorem 3.3 Let $f$ be a non-constant meromorphic function of zero order, let $q \in \mathbb{C} \backslash\{0,1\}$, and let $a_{1}, \ldots, a_{p}, p \geq 2$, be distinct constants. Then

$$
(p-1) T(r, f) \leq \widetilde{N}_{q}(r, f)+\sum_{k=1}^{p} \widetilde{N}_{q}\left(r, \frac{1}{f-a_{k}}\right)+S_{q}(r, f) .
$$

The definition of $\widetilde{N}_{q}(r, a)$ alone gives no obvious lower bound for $\widetilde{N}_{q}(r, a)$. However, Theorem 3.3 implies that the inequality $\widetilde{N}_{q}(r, a) \geq-T(r, f)+o(T(r, f))$ holds, for any $a \in \mathbb{C} \cup\{\infty\}$ and for all $r$ outside of a possible exceptional set $E$ with zero logarithmic density.

A difference analogue of the index of multiplicity $\theta(a, f)$ is called the $q$ separated pair index, and it is defined as follows:

$$
\pi_{q}(a, f):=\liminf _{r \rightarrow \infty} \frac{N_{q}(r, a)}{T(r, f)}
$$

where $a \in \mathbb{C} \cup\{\infty\}$. Similarly, we define

$$
\Pi_{q}(a, f):=1-\limsup _{r \rightarrow \infty} \frac{\widetilde{N}_{q}(r, a)}{T(r, f)},
$$

which is an analogue of $\Theta(a, f)$, see [15]. Now we have the necessary notation to write down the following consequence of Theorem 3.3.

Corollary 3.4 Let $q \in \mathbb{C} \backslash\{0,1\}$, and let $f$ be a non-constant meromorphic function of zero order. Then $\Pi_{q}(a, f)=0$ except for at most countably many values $a$, and

$$
\sum_{a}\left(\delta(a, f)+\pi_{q}(a, f)\right) \leq \sum_{a} \Pi_{q}(a, f) \leq 2 .
$$

In the next section we will give an explicit example of a zero-order meromorphic function $g$ having a large number of zeros and poles, but nevertheless satisfying

$$
\Pi_{q}(\infty, g)+\Pi_{q}(0, g)=2 .
$$

Analogously to complete ramification, we say that a point $a$ is completely paired with the separation $q$ if whenever $f(z)=a$ then either $f(q z)=a$ or $f\left(q^{-1} z\right)=a$, with the same multiplicity. Then a non-constant meromorphic function of zero order can have at most four values which only appear in pairs.

Corollary 3.5 Let $q \in \mathbb{C} \backslash\{0,1\}$, and let $f$ be a non-constant meromorphic function of zero order. Then $f$ has at most four completely paired points with separation $q$. 
Another consequence of Nevanlinna's Second Main Theorem is the five value theorem, which says that if two non-constant meromorphic functions share five values ignoring multiplicity then these functions must be identical. We say that two meromorphic functions $f$ and $g$ share a point a, ignoring $q$-separated pairs, when $f(z)=a$ if and only if $g(z)=a$ with the same multiplicity, unless $a$ is a $q$-separated pair of $f$ or $g$. In other words, all paired points are ignored when determining whether or not $f$ and $g$ share $a$.

Theorem 3.6 Let $q \in \mathbb{C} \backslash\{0,1\}$, and let $f$ and $g$ be meromorphic functions of zero order. If there are five distinct points $a_{k} \in \mathbb{C} \cup\{\infty\}$ such that $f$ and $g$ share $a_{k}$, ignoring $q$-separated pairs, for all $k=1, \ldots, 5$ then either $f(z) \equiv g(z)$ or both $f$ and $g$ are constants.

\section{The $q$-Gamma Function and $q$-Difference Equa- tions}

In this section we illustrate some of the results obtained in previous sections with some examples of $q$-difference equations which are explicitly solvable in terms of known zero-order meromorphic functions. Let $q \in \mathbb{C}$ be such that $0<|q|<1$. Then the $q$-Gamma function $\Gamma_{q}(x)$ is defined by

$$
\Gamma_{q}(x):=\frac{(q ; q)_{\infty}}{\left(q^{x} ; q\right)_{\infty}}(1-q)^{1-x}
$$

where $(a ; q)_{\infty}=\Pi_{k=0}^{\infty}\left(1-a q^{k}\right)$. It is a meromorphic function with poles at $x=-n \pm 2 \pi i k / \log q$, where $k$ and $n$ are non-negative integers [1]. By defining

$$
\gamma_{q}(z):=(1-q)^{x-1} \Gamma_{q}(x), \quad z=q^{x},
$$

and $\gamma_{q}(0):=(q ; q)_{\infty}$, we have that $\gamma_{q}(z)$ is a meromorphic function of zero order with no zeros, having its poles at $\left\{q^{-k}\right\}_{k=0}^{\infty}$. Therefore poles and zeros of $\gamma_{q}(z)$ are $q$-Picard exceptional, and $\gamma_{q}(z)$ cannot have any other exceptional values by Corollary 3.2. Similarly, for $z_{0} \in \mathbb{C}$, the zeros and poles of the function

$$
g_{q}(z):=\gamma_{q}(z) / \gamma_{q}\left(z-z_{0}\right),
$$

are at $\left\{z_{0}+q^{-k}\right\}_{k=0}^{\infty}$ and $\left\{q^{-k}\right\}_{k=0}^{\infty}$, respectively. Hence, if $z_{0}$ is chosen such that $z_{0} \notin\left\{q^{-k}\right\}_{k=0}^{\infty}$, then $g_{g}(z)$ is a non-rational meromorphic function of zero order having the maximal two $q$-Picard exceptional values, but no (ordinary) Picard exceptional values (by Corollary 3.2). Moreover,

$$
\Pi_{q}\left(0, g_{q}\right)+\Pi_{q}\left(\infty, g_{q}\right)=2
$$

which shows that upper bound in (13) may be attained with two non-deficient values in the usual sense.

The first-order linear $q$-difference equation

$$
f(q z)=(1-z) f(z)
$$


is satisfied by the function $\gamma_{q}(z)$. Consider now the general first-order linear $q$-difference equation

$$
f(q z)=a(z) f(z),
$$

where the coefficient $a(z)$ is a rational function. If $a(z) \equiv a$ is constant, equation (14) is solvable in terms of rational functions if and only if $\log _{q} a$ is an integer. If $a(z)$ is non-constant, let $\alpha_{i}, i=1, \ldots, n$, and $\beta_{j}, j=1, \ldots, m$, be the zeros and poles of $a(z)$, respectively, repeated according to their multiplicity. Then $a(z)$ can be written in the form

$$
a(z)=\frac{c\left(1-z / \alpha_{1}\right) \cdots\left(1-z / \alpha_{n}\right)}{\left(1-z / \beta_{1}\right) \cdots\left(1-z / \beta_{m}\right)}
$$

where $c \neq 0$ is a complex number depending on $a(z)$. Therefore, equation (14) is solved by

$$
f(z)=z^{\log _{q} c} \frac{\gamma_{q}\left(z / \alpha_{1}\right) \cdots \gamma_{q}\left(z / \alpha_{n}\right)}{\gamma_{q}\left(z / \beta_{1}\right) \cdots \gamma_{q}\left(z / \beta_{m}\right)}
$$

which is meromorphic if and only if $\log _{q} c$ is an integer.

Now consider the second order linear $q$-difference equation

$$
f\left(q^{2} z\right)+a_{1}(z) f(q z)+a_{0}(z) f(z)=0,
$$

where $a_{1}(z)$ and $a_{0}(z) \not \equiv 0$ are rational functions. By [3] all meromorphic solutions $f(z)$ of $(15)$ satisfy $T(r, f)=O\left((\log r)^{2}\right)$. Also, if equation (15) has a meromorphic solution $f(z)$, then $g(z)=f(q z) / f(z)$ is a solution of the $q$ difference Riccati equation

$$
g(q z)=-\frac{a_{1}(z) g(z)+a_{0}(z)}{g(z)} .
$$

Since $f(z)$ is of order zero, so is $g(z)$, and hence we may apply Theorem 2.1 with $P(z, q)=g(z)$ and $Q(z, g)=-\left(a_{1}(z) g(z)+a_{0}(z)\right)$ to obtain

$$
m(r, g)=o\left(T\left(r, g\left(q^{-1} z\right)\right)\right)=o\left(T\left(\left|q^{-1}\right| r, g\right)\right)
$$

for all $r$ on a set with logarithmic density 1 . Therefore, $m(r, g)=o(T(r, g))$ by [16, Lemma 4] (see also Lemma B below), and so if $g(z)$ is non-rational, its poles are non-deficient in the sense that the Nevanlinna deficiency satisfies $\delta(\infty, g)=0$. In particular, it follows that $g(z)$ has infinitely many poles.

\section{$5 \quad$ Proof of Theorem 1.1}

As the proof of the Lemma on the Logarithmic Derivative [5, 15, 21] the proof of the following lemma relies on the Poisson-Jensen Formula. 
Lemma 5.1 Let $f$ be a meromorphic function such that $f(0) \neq 0, \infty$ and let $q \in \mathbb{C} \backslash\{0\}$. Then,

$$
\begin{aligned}
m\left(r, \frac{f(q z)}{f(z)}\right) \leq & \left(n(\rho, f)+n\left(\rho, \frac{1}{f}\right)\right)\left(\frac{|q-1|^{\delta}\left(|q|^{\delta}+1\right)}{\delta(\delta-1)|q|^{\delta}}+\frac{|q-1| r}{\rho-|q| r}+\frac{|q-1| r}{\rho-r}\right) \\
& +\frac{4|q-1| r \rho}{(\rho-r)(\rho-|q| r)}\left(T(\rho, f)+\log ^{+}\left|\frac{1}{f(0)}\right|\right),
\end{aligned}
$$

where $z=r e^{i \phi}, \rho>\max \{r,|q| r\}$ and $0<\delta<1$.

Proof. Using the identity

$$
\frac{\rho^{2}-r^{2}}{\rho^{2}-2 \rho r \cos (\varphi-\theta)+r^{2}}=\operatorname{Re}\left(\frac{\rho e^{i \theta}+z}{\rho e^{i \theta}-z}\right), \quad z=r e^{i \varphi},
$$

and the Poisson-Jensen formula [15] with $R=\rho$ we see

$$
\begin{aligned}
\log \left|\frac{f(q z)}{f(z)}\right|= & \int_{0}^{2 \pi} \log \left|f\left(\rho e^{i \theta}\right)\right| \operatorname{Re}\left(\frac{2 \rho z e^{i \theta}(q-1)}{\left(\rho e^{i \theta}-z\right)\left(\rho e^{i \theta}-q z\right)}\right) \frac{d \theta}{2 \pi} \\
& +\sum_{\left|a_{n}\right|<\rho} \log \left|\frac{\left(q z-a_{n}\right)\left(\rho^{2}-\bar{a}_{n} z\right)}{\left(z-a_{n}\right)\left(\rho^{2}-\bar{a}_{n} q z\right)}\right| \\
& -\sum_{\left|b_{m}\right|<\rho} \log \left|\frac{\left(q z-b_{m}\right)\left(\rho^{2}-\bar{b}_{m} z\right)}{\left(z-b_{m}\right)\left(\rho^{2}-\bar{b}_{m} q z\right)}\right| \\
=: & S_{1}(z)+S_{2}(z)-S_{3}(z),
\end{aligned}
$$

where $\left\{a_{n}\right\}$ and $\left\{b_{m}\right\}$ are the zeros and poles of $f$ respectively. Integration on the set $E:=\left\{\psi \in[0,2 \pi]:\left|\frac{f\left(q r e^{i \psi}\right)}{f\left(r e^{i \psi}\right)}\right| \geq 1\right\}$ gives us the proximity function,

$$
\begin{aligned}
m\left(r, \frac{f(q z)}{f(z)}\right) & =\int_{E} \log \left|\frac{f(q z)}{f(z)}\right| \frac{d \psi}{2 \pi} \\
& =\int_{E}\left(S_{1}\left(r e^{i \psi}\right)+S_{2}\left(r e^{i \psi}\right)-S_{3}\left(r e^{i \psi}\right)\right) \frac{d \psi}{2 \pi} \\
& \leq \int_{0}^{2 \pi}\left(\left|S_{1}\left(r e^{i \psi}\right)\right|+\left|S_{2}\left(r e^{i \psi}\right)\right|+\left|S_{3}\left(r e^{i \psi}\right)\right|\right) \frac{d \psi}{2 \pi} .
\end{aligned}
$$

We will now proceed to estimate each $\int_{0}^{2 \pi}\left|S_{j}\left(r e^{i \psi}\right)\right| \frac{d \psi}{2 \pi}$ separately. Since

$$
\begin{aligned}
\left|S_{1}(z)\right| & =\left|\frac{1}{2 \pi} \int_{0}^{2 \pi} \log \right| f\left(\rho e^{i \theta}\right)\left|\operatorname{Re}\left(\frac{2(q-1) z \rho e^{i \theta}}{\left(\rho e^{i \theta}-q z\right)\left(\rho e^{i \theta}-z\right)}\right) d \theta\right| \\
& \leq\left|\frac{1}{2 \pi} \frac{2 \rho(q-1) r}{(\rho-|q| r)(\rho-r)}\right| \int_{0}^{2 \pi}|\log | f\left(\rho e^{i \theta}\right)|| d \theta \\
& \leq\left|\frac{2 \rho(q-1) r}{(\rho-|q| r)(\rho-r)}\right|\left(m(\rho, f)+m\left(\rho, \frac{1}{f}\right)\right),
\end{aligned}
$$


we have

$$
\int_{0}^{2 \pi}\left|S_{1}\left(r e^{i \psi}\right)\right| \frac{d \psi}{2 \pi} \leq \frac{4 \rho|q-1| r}{(\rho-|q| r)(\rho-r)}\left(T(\rho, f)+\log ^{+} \frac{1}{|f(0)|}\right) .
$$

By denoting $\left\{c_{n}\right\}=\left\{a_{n}\right\} \cup\left\{b_{n}\right\}$ we may consider the integrals of $S_{2}$ and $S_{3}$ simultaneously. Then,

$$
\begin{aligned}
& \int_{0}^{2 \pi}\left(\left|S_{2}\left(r e^{i \psi}\right)\right|+\left|S_{3}\left(r e^{i \psi}\right)\right|\right) \frac{d \psi}{2 \pi} \leq \sum_{\left|c_{n}\right|<\rho} \int_{0}^{2 \pi}|\log | \frac{\left(q r e^{i \psi}-c_{n}\right)\left(\rho^{2}-\bar{c}_{n} r e^{i \psi}\right)}{\left(r e^{i \psi}-c_{n}\right)\left(\rho^{2}-\bar{c}_{n} q r e^{i \psi}\right)}|| \frac{d \psi}{2 \pi} \\
& \leq \sum_{\left|c_{n}\right|<\rho} \int_{0}^{2 \pi} \log ^{+}\left|1+\frac{(q-1) \bar{c}_{n} r e^{i \psi}}{\rho^{2}-\bar{c}_{n} q r e^{i \psi}}\right| \frac{d \psi}{2 \pi}+\sum_{\left|c_{n}\right|<\rho} \int_{0}^{2 \pi} \log ^{+}\left|1-\frac{(q-1) \bar{c}_{n} r e^{i \psi}}{\rho^{2}-\bar{c}_{n} r e^{i \psi}}\right| \frac{d \psi}{2 \pi} \\
& \quad+\sum_{\left|c_{n}\right|<\rho} \int_{0}^{2 \pi} \log ^{+}\left|1+\frac{(q-1) r e^{i \psi}}{r e^{i \psi}-c_{n}}\right| \frac{d \psi}{2 \pi}+\sum_{\left|c_{n}\right|<\rho} \int_{0}^{2 \pi} \log ^{+}\left|1-\frac{(q-1) r e^{i \psi}}{q r e^{i \psi}-c_{n}}\right| \frac{d \psi}{2 \pi}
\end{aligned}
$$

Using the fact that $\log (1+|x|) \leq|x|$ for all $x$ we have

$$
\begin{aligned}
\int_{0}^{2 \pi} \log ^{+}\left|1+\frac{(q-1) r e^{i \psi}}{r e^{i \psi}-c_{n}}\right| \frac{d \psi}{2 \pi} & \leq \frac{1}{\delta} \int_{0}^{2 \pi} \log ^{+}\left(1+\left|\frac{(q-1) r e^{i \psi}}{r e^{i \psi}-c_{n}}\right|\right)^{\delta} \frac{d \psi}{2 \pi} \\
& \leq \frac{1}{\delta} \int_{0}^{2 \pi}\left|\frac{r e^{i \psi}(q-1)}{r e^{i \psi}-c_{n}}\right|^{\delta} \frac{d \psi}{2 \pi} \\
& =\frac{|q-1|^{\delta} r^{\delta}}{2 \pi \delta} \int_{0}^{2 \pi} \frac{d \psi}{\left|r e^{i \psi}-\right| c_{n}||^{\delta}} .
\end{aligned}
$$

Then using $\left|r e^{i \psi}-\right| c_{n}||>\frac{2}{\pi} r \psi$ for all $0 \leq \psi \leq \frac{\pi}{2}$ we get

$$
\begin{aligned}
\int_{0}^{2 \pi} \log ^{+}\left|1+\frac{(q-1) r e^{i \psi}}{r e^{i \psi}-c_{n}}\right| \frac{d \psi}{2 \pi} & \leq \frac{4|q-1|^{\delta} r^{\delta}}{2 \pi \delta} \int_{0}^{\frac{\pi}{2}} \frac{d \psi}{\left.\left|r e^{i \psi}-\right| c_{n}\right|^{\delta}} \\
& \leq \frac{4|q-1|^{\delta} r^{\delta}}{2 \pi \delta} \int_{0}^{\frac{\pi}{2}} \frac{d \psi}{\left(\frac{2}{\pi} r \psi\right)^{\delta}} \\
& =\frac{|q-1|^{\delta}}{\delta(1-\delta)}
\end{aligned}
$$

and

Also, we have

$$
\int_{0}^{2 \pi} \log ^{+}\left|1-\frac{(q-1) r e^{i \psi}}{q r e^{i \psi}-c_{n}}\right| \frac{d \psi}{2 \pi} \leq \frac{|q-1|^{\delta}}{|q|^{\delta} \delta(1-\delta)} .
$$

$$
\begin{aligned}
\int_{0}^{2 \pi} \log ^{+}\left|1+\frac{(q-1) \bar{c}_{n} r e^{i \psi}}{\rho^{2}-\bar{c}_{n} q r e^{i \psi}}\right| \frac{d \psi}{2 \pi} & \leq \int_{0}^{2 \pi} \log ^{+}\left(1+\left|\frac{(q-1) \bar{c}_{n} r e^{i \psi}}{\rho^{2}-\bar{c}_{n} q r e^{i \psi}}\right|\right) \frac{d \psi}{2 \pi} \\
& \leq \int_{0}^{2 \pi}\left|\frac{(q-1) \bar{c}_{n} r e^{i \psi}}{\rho^{2}-\bar{c}_{n} q r e^{i \psi}}\right| \frac{d \psi}{2 \pi} \\
& =|q-1| r \int_{0}^{2 \pi}\left|\frac{c_{n}}{\rho^{2}-\bar{c}_{n} q r e^{i \psi}}\right| \frac{d \psi}{2 \pi}
\end{aligned}
$$


Using the fact that for all $a$ such that $|a|<\rho$,

$$
\left|\frac{a}{\rho^{2}-\bar{a} r e^{i \psi}}\right| \leq \frac{1}{\rho-r}
$$

we obtain

$$
\int_{0}^{2 \pi} \log ^{+}\left|1+\frac{(q-1) \bar{c}_{n} r e^{i \psi}}{\rho^{2}-\bar{c}_{n} q r e^{i \psi}}\right| \frac{d \psi}{2 \pi} \leq \frac{|q-1| r}{\rho-|q| r},
$$

and

$$
\int_{0}^{2 \pi} \log ^{+}\left|1-\frac{(q-1) \bar{c}_{n} r e^{i \psi}}{\rho^{2}-\bar{c}_{n} z}\right| \frac{d \psi}{2 \pi} \leq \frac{|q-1| r}{\rho-r} .
$$

Combining (16), (17), (18) and (19) gives

$$
\begin{aligned}
& \int_{0}^{2 \pi}\left(\left|S_{2}\right|+\left|S_{3}\right|\right) \frac{d \psi}{2 \pi} \\
& \quad \leq\left(n(\rho, f)+n\left(\rho, \frac{1}{f}\right)\right)\left(\frac{|q-1|^{\delta}\left(|q|^{\delta}+1\right)}{\delta(1-\delta)|q|^{\delta}}+\frac{|q-1| r}{\rho-|q| r}+\frac{|q-1| r}{\rho-r}\right) .
\end{aligned}
$$

The assertion follows by combining the obtained bounds for the $S_{i}$ terms.

If $f(z)$ has either a zero or a pole at the origin, then, for a suitable $p \in \mathbb{Z}$, we may write $f(z)=z^{p} g(z)$ where $g(z)$ is finite and non-zero at the origin. Hence, by taking $K>1$ and applying Lemma 5.1 with $\rho=K r$, we have, for all $r$ sufficiently large,

$$
m\left(r, \frac{f(q z)}{f(z)}\right) \leq D_{1}\left(n(K r, f)+n\left(K r, \frac{1}{f}\right)\right)+\frac{D_{2}}{K} T(K r, f)
$$

where $D_{1}$ and $D_{2}$ are constants independent of $r$ and $K$.

In order to deal with the $T(K r, f)$ term we use the following result which is a special case of [16, Lemma 4].

Lemma B If $T: \mathbb{R}^{+} \rightarrow \mathbb{R}^{+}$is an increasing function such that

$$
\varlimsup_{r \rightarrow \infty} \frac{\log T(r)}{\log r}=0,
$$

then the set

$$
E:=\left\{r: T\left(C_{1} r\right) \geq C_{2} T(r)\right\}
$$

has logarithmic density 0 for all $C_{1}>1$ and $C_{2}>1$.

To show that the $n(K r, f)$ term in equation (20) is small we first prove the following lemma.

Lemma 5.2 If $f$ is a non-constant meromorphic function of zero order, then the set

$$
E_{n}:=\left\{r \geq 1: n(r, f)<\frac{T(r, f)}{2^{n}}\right\}
$$

has logarithmic density 1 for all $n \in \mathbb{N}$. 
Proof. Since, by Lemma B, $N(K r, f) \leq 2 N(r, f)$ on a set of logarithmic density 1 for any $K>1$, and $N(K r, f) \geq n(r, f) \log K$ for all $r \geq 1$, it follows that

$$
n(r, f) \leq \frac{1}{\log K} N(K r, f) \leq \frac{2}{\log K} N(r, f) \leq \frac{2}{\log K} T(r, f)
$$

on a set of logarithmic density 1 . By choosing $K>\exp 2^{n+1}$ we have that the set

$$
E_{n}:=\left\{r \geq 1: n(r, f)<\frac{T(r, f)}{2^{n}}\right\}
$$

has logarithmic density 1 for all $n \in \mathbb{N}$, as required.

The following lemma, together with Lemma 5.2, implies that $n(r, f)=$ $o(T(r, f))$ on a set of logarithmic density 1 , whenever $f$ is a meromorphic function with zero order.

Lemma 5.3 Let $T: \mathbb{R}^{+} \rightarrow \mathbb{R}^{+}$be an increasing function, and let $U: \mathbb{R}^{+} \rightarrow \mathbb{R}^{+}$. If there exists a decreasing sequence $\left\{c_{n}\right\}_{n \in \mathbb{N}}$ such that $c_{n} \rightarrow 0$ as $n \rightarrow \infty$, and, for all $n \in \mathbb{N}$, the set

$$
F_{n}:=\left\{r \geq 1: U(r)<c_{n} T(r)\right\}
$$

has logarithmic density 1, then

$$
U(r)=o(T(r))
$$

on a set of logarithmic density 1.

Proof. Since each set $F_{n}$ has logarithmic density 1 we have

$$
\varlimsup_{r \rightarrow \infty} \frac{1}{\log r} \int_{[1, r] \cap F_{n}} \frac{d t}{t}=1,
$$

which implies that for all $n$, there exists $r_{n}$, such that

$$
\frac{1}{\log r} \int_{[1, r] \cap F_{n}} \frac{d t}{t}>1-\frac{1}{n}, \quad \text { for all } r \geq r_{n} .
$$

We set $F$ to be the union of the sets $\left[r_{n}, r_{n+1}\right) \cap F_{n}$ where $n$ runs through all positive integers. Then for all $r \in F$ we have that

$$
U(r)<c_{n_{r}} T(r),
$$

where $n_{r} \rightarrow \infty$ as $r \rightarrow \infty$. Since $c_{n} \rightarrow 0$ as $n \rightarrow \infty$, this implies that $U(r)=$ $o(T(r, f))$ on $F$. Therefore if we can show that $F$ has logarithmic density 1 we are done.

Since for all sufficiently large $r$ there is $n$ so that $r_{n} \leq r \leq r_{n+1}$, we have

$$
\int_{[1, r] \cap F} \frac{d t}{t} \geq \int_{[1, r] \cap F_{n}} \frac{d t}{t}>\left(1-\frac{1}{n}\right) \log r
$$


Dividing through by $\log r$ and taking the limit as $n \rightarrow \infty$ gives us that $F$ has logarithmic density 1 , as required.

The following corollary is an immediate consequence of Lemmas 5.2 and 5.3.

Corollary 5.4 If $f$ is a non-constant meromorphic function of zero order, then

$$
n(r, f)=o(T(r, f))
$$

on a set of logarithmic density 1 .

Now we are ready to complete the proof of Theorem 1.1. By Lemma B we have that $T(K r, f) \leq 2 T(r, f)$ and $n(K r, f)+n(K r, 1 / f) \leq 2(n(r, f)+n(r, 1 / f))$ on a set of logarithmic density 1 , and so, by (20),

$$
m\left(r, \frac{f(q z)}{f(z)}\right) \leq 2 D_{1}\left(n(r, f)+n\left(r, \frac{1}{f}\right)\right)+\frac{2 D_{2}}{K} T(r, f)
$$

for all $r$ on a set with logarithmic density 1 . Therefore, by choosing $K=2^{n}$, and by applying Lemma 5.2, we have

$$
m\left(r, \frac{f(q z)}{f(z)}\right)<\frac{4 D_{1}+2 D_{2}}{2^{n}} T(r, f)
$$

on a set with logarithmic density 1 for all $n \in \mathbb{N}$. By taking

$$
U(r):=m\left(r, \frac{f(q z)}{f(z)}\right)
$$

in Lemma 5.3, we obtain

$$
m\left(r, \frac{f(q z)}{f(z)}\right)=o(T(r, f))
$$

on a set of logarithmic density 1 , as required.

\section{Conclusion}

In this paper we have extended some of the main results of Nevanlinna theory to the $q$-difference operator acting on zero-order meromorphic functions. In particular, we have found $q$-difference analogues of the Lemma on the Logarithmic Derivative (see Theorem 1.1) and the Second Main Theorem (see Theorem 3.1). The $q$-difference analogue of the Second Main Theorem implies, for instance, that if a zero-order meromorphic function $f$ assumes three distinct values $a, b$ and $c$ only in infinite point sets $\left\{q^{n} z_{j}\right\}_{n \in \mathbb{N} \cup\{0\}}$, then $f$ must be constant. This is a $q$-shift analogue of Picard's theorem. Our findings are an analogue of the results concerning the difference operator by Halburd and Korhonen in [13, 14].

Historically $q$-difference equations are one of the most natural classes of equations to look at after differential equations and difference equations, and 
the study of meromorphic solutions of $q$-difference equations has been ongoing since the nineteenth century. As seen in Section 2, the $q$-shift analogue of the Lemma on the Logarithmic Derivative enables an efficient study of the value distribution of zero-order meromorphic solutions of large classes of $q$ difference equations. Theorem 2.1 is a powerful tool when analyzing densities of poles of solutions, while Theorem 2.2 can be used to obtain information on the value distribution of almost any finite value. The restriction to zero-order meromorphic solutions is natural in the sense that all meromorphic solutions of linear $q$-difference equations and $q$-difference Riccati equations have zero order $[3,10]$.

Acknowledgments. We wish to thank Professor J. K. Langley for valuable discussions. In particular for showing that for any zero-order meromorphic function $f$, we have $n(r, f)=o(T(r, f))$ on a set of logarithmic density one. The research reported in this paper was supported in part by the Academy of Finland (grant no: 204819), the European Commission's Framework 6 ENIGMA Network and the Leverhulme Trust.

\section{References}

[1] G. E. Andrews, R. Askey, and R. Roy. Special Functions. Cambridge University Press, Cambridge, 1999.

[2] W. Bergweiler and W. Hayman. Zeros of solutions of a functional equation. Comput. Methods Funct. Theory, 3:55-78, 2003.

[3] W. Bergweiler, K. Ishizaki, and N. Yanagihara. Meromorphic solutions of some functional equations. Methods Appl. Anal., 5:248-258, 1998. Correction: Methods Appl. Anal. 6, 1999.

[4] W. Bergweiler, K. Ishizaki, and N. Yanagihara. Growth of meromorphic solutions of some functional equations. I. Aequationes Math., 63:140-151, 2002 .

[5] W. Cherry and Z. Ye. Nevanlinna's theory of value distribution. SpringerVerlag, Berlin, 2001.

[6] Y. M. Chiang and S. J. Feng. On the Nevanlinna characteristic of $f(z+\eta)$ and difference equations in the complex plane. preprint, 2005.

[7] J. Clunie. On integral and meromorphic functions. J. London Math. Soc., $37: 17-27,1962$.

[8] A. E. Eremenko and M. L. Sodin. Iterations of rational functions and the distribution of the values of Poincaré functions. Teor. Funktsii Funktsional. Anal. i Prilozhen., 53:18-25, 1990. (Russian); translation in J. Soviet Math. 58 (1992), no. 6, 504-509. 
[9] V. I. Gromak, I. Laine, and S. Shimomura. Painlevé differential equations in the complex plane. Walter de Gruyter, Berlin, 2002.

[10] G. G. Gundersen, J. Heittokangas, I. Laine, J. Rieppo, and D. Yang. Meromorphic solutions of generalized Schröder equations. Aequationes Math., 63:110-135, 2002.

[11] R. G. Halburd and R. J. Korhonen. Existence of finite order meromorphic solutions as a detector of integrability in difference equations. Preprint, 2005 .

[12] R. G. Halburd and R. J. Korhonen. Finite-order meromorphic solutions and the discrete Painlevé equations. to appear in Proc. London Math. Soc., http://arXiv.org, no: nlin.SI/0504026.

[13] R. G. Halburd and R. J. Korhonen. Difference analogue of the lemma on the logarithmic derivative with applications to difference equations. $J$. Math. Anal. Appl., 314:477-487, 2006.

[14] R. G. Halburd and R. J. Korhonen. Nevanlinna theory for the difference operator. to appear in Ann. Acad. Sci. Fenn. Math., http://arXiv.org, no: math.CV/0506011.

[15] W. K. Hayman. Meromorphic functions. Clarendon Press, Oxford, 1964.

[16] W. K. Hayman. On the characteristic of functions meromorphic in the plane and of their integrals. Proc. London Math. Soc., 14a:93-128, 1965.

[17] J. Heittokangas, I. Laine, J. Rieppo, and D. Yang. Meromorphic solutions of some linear functional equations. Aequationes Math., 60:148-166, 2000.

[18] K. Ishizaki and N. Yanagihara. Deficiency for meromorphic solutions of Schröder equations. Complex Var. Theory Appl., 49:539-548, 2004.

[19] K. Ishizaki and N. Yanagihara. Borel and Julia directions of meromorphic Schröder functions. Math. Proc. Camb. Phil. Soc., 139:139-147, 2005.

[20] K. Ishizaki and N. Yanagihara. Remarks on deficiencies for meromorphic Schröder functions. to appear in Advanced Studies of Pure Mathematics, XX, 2005, Potential Theory in Matsue, 2005.

[21] G. Jank and L. Volkmann. Einführung in die Theorie der ganzen und meromorphen Funktionen mit Anwendungen auf Differentialgleichungen. Birkhäuser Verlag, Basel, 1985.

[22] I. Laine. Nevanlinna theory and complex differential equations. Walter de Gruyter, Berlin, 1993.

[23] J. Malmquist. Sur les fonctions á un nombre fini des branches définies par les équations différentielles du premier ordre. Acta Math., 36:297-343, 1913. 
[24] A. Z. Mohon'ko and V. D. Mohon'ko. Estimates of the Nevanlinna characteristics of certain classes of meromorphic functions, and their applications to differential equations. Sibirsk. Mat. Zh., 15:1305-1322, 1974. (Russian).

[25] R. Nevanlinna. Zur Theorie der meromorphen Funktionen. Acta Math., 46:1-99, 1925.

[26] J.-P. Ramis. About the growth of entire functions solutions of linear algebraic q-difference equations. Ann. Fac. Sci. Toulouse Math., 1:53-94, 1992.

[27] G. Valiron. Fonctions Analytiques. Press. Univ. de France, Paris, 1952. 